\title{
COMPORTAMENTO REOLÓGICO E NOMENCLATURA DOS ALIMENTOS UTILIZADOS POR FONOAUDIÓLOGOS DE SERVIÇOS DE DISFAGIA
}

\author{
Rheological behavior and labels of texture-modified foods \\ and thickened fluids as used for dysphasia's services
}

\author{
Marina de Sordi (1), Lúcia Figueiredo Mourão (2), Luciano Bruno de Carvalho Silva ${ }^{(3)}$
}

\begin{abstract}
RESUMO
Objetivo: estudar o comportamento reológico e a forma de classificação e nomeação das diferentes preparações utilizadas nas intervenções com pacientes disfágicos, realizada por fonoaudiólogos do estado de São Paulo, tendo como referência a proposta australiana de padronização da nomenclatura. Método: estudo quantitativo descritivo e qualitativo. Os dados foram coletados por meio da aplicação de questionários semi-estruturados com fonoaudiólogos de serviços de disfagia no estado de São Paulo. Os fonoaudiólogos relataram os alimentos utilizados em sua prática por meio de exemplos de preparações bem como exemplos comerciais. Os profissionais classificaram os alimentos em diferentes níveis e relataram a nomenclatura utilizada para cada nível. A viscosidade das preparações foi medida em viscosímetro e agrupadas de acordo com os valores obtidos. Em seguida foram classificados de acordo a proposta australiana de unificação da nomenclatura. Resultados: foram analisados 18 questionários. Os profissionais utilizaram alimentos de comportamento reológico próximo em sua prática. Os fonoaudiólogos apresentaram, em sua maioria, 06 formas de agrupamento dos alimentos baseados em suas características reológicas, principalmente a viscosidade. Foram apontados 33 termos para nomear 07 classes de alimentos segundo a proposta australiana. Os principais termos foram: líquido, pastoso e sólido, seguidos de graduações e especificações. Conclusão: fonoaudiólogos de diferentes serviços de atenção em disfagia utilizam diferentes terminologias para designar a mesma preparação no estado de São Paulo. São necessários maiores estudos para que se possa estabelecer uma nomenclatura unificada no Brasil.
\end{abstract}

DESCRITORES: Transtornos de Deglutição; Reologia; Comunicação Interdisciplinar

\section{INTRODUÇÃO}

Atualmente são freqüentes os estudos na literatura que analisam as características reológicas dos alimentos e sua influência no processo de

(1) Fonoaudióloga clínica; Mestre em Saúde Interdisciplinaridade e Reabilitação pela Universidade Estadual de Campinas - UNICAMP.

(2) Fonoaudióloga; Docente da Universidade Estadual de Campinas -Unicamp,Campinas, SP, Brasil; Doutora em Ciências pela Universidade Federal de São Paulo UNIFESP.

(3) Nutricionista; Professor Adjunto da Faculdade de Nutrição da Universidade Federal de Alfenas - Unifal-MG, Alfenas, MG, Brasil; Doutor em Alimentos e Nutrição pela Universidade Estadual de Campinas.

Conflito de interesses: inexistente deglutição ${ }^{1-3}$. A análise do comportamento reológico dos alimentos permite utilizar características específicas (elasticidade, densidade, mastigabilidade, viscosidade e textura) como parâmetros de classificação dos mesmos em níveis pré-determinados.

A viscosidade é uma das características reológicas de um fluido e é definida como a taxa de fluxo por unidade de força, ou seja, é a resistência, o atrito, de um fluido ao fluxo. A viscosidade pode ser selecionada como um dos parâmetros de classificação, uma vez que é possível mensurá-la e classificá-la em centipoises (gramas/centímetro/ segundo) nos diferentes tipos de alimentos ${ }^{4}$.

Classificar alimentos segundo parâmetros reológicos específicos, (viscosidade, por exemplo) permite estabelecer padrões que possibilitam a 
classificação de diferentes preparações em níveis pré-determinados, sendo assim possível nomeá-los de forma similar ${ }^{5}$.

A padronização de uma nomenclatura para se referir aos alimentos frequentemente utilizados nas intervenções com pacientes disfágicos tornase fundamental visto que dentre as estratégias para reabilitação do paciente enquadram-se alterações na viscosidade da dieta ${ }^{6}$. Ao promoverem mudanças no padrão de deglutição, tais mudanças podem proporcionar uma ingestão segura e eficaz ao paciente ${ }^{7-10}$.

Prescrições relacionadas a alterações na viscosidade de líquidos e textura da dieta, que comumente ocorrem entre profissionais que atuam em equipes de intervenção em disfagia, são imprescindíveis na reabilitação do paciente. Interpretações errôneas de laudos e prescrições podem colocar em risco não apenas o processo de habilitação/ reabilitação do paciente como também sua vida. $\mathrm{A}$ falta de uma terminologia em comum para designar as diferentes alterações na dieta promove risco ao processo de reabilitação gerando dúvidas e mal entendidos entre profissionais.

No Brasil não se encontra terminologia unificada disponível na literatura para classificar as diversas alterações na dieta utilizadas nas intervenções com pacientes disfágicos. Países como Reino Unido, Estados Unidos e Austrália estabeleceram padrões de nomenclatura dos alimentos.

A classificação utilizada no Reino Unido ${ }^{11}$ propõe quarto divisões para líquidos engrossados: líquidos naturalmente engrossados, líquidos engrossados fase 1, líquidos engrossados fase 2 e líquidos engrossados fase 3 e cinco para sólidos (textura A, B, C, D, E). A proposta americana ${ }^{12}$ baseia sua padronização de líquidos engrossados na viscosidade dos mesmos e os divide em: líquido (10 $50 \mathrm{cP})$, néctar (51-350cP), mel (350 - 1750cP) e pudim (acima de $1750 \mathrm{cP}$ ), apresentando ainda três divisões para sólidos (entre 1 e $2,5 \mathrm{~cm} ; 0,6 \mathrm{~cm}$ e purê).

A proposta australiana de padronização ${ }^{13}$ apresenta três níveis de classificação para líquidos engrossados e três níveis para alimentos sólidos, acompanha ainda uma grande lista de exemplos de alimentos para cada nível de classificação proposto. Essa proposta foi selecionada como base para as análises do presente estudo por apresentar exemplos de alimentos que são comuns na realidade brasileira.

Assim, considerando tais questões, o objetivo deste estudo é estudar o comportamento reológico e a forma de classificação e nomeação das principais preparações utilizados nas intervenções com pacientes disfágicos, realizada por fonoaudiólogos do estado de São Paulo - Brasil, tendo como referência a proposta australiana de padronização da nomenclatura.

\section{MÉTODO}

Foram convidados a participar do estudo 30 profissionais de fonoaudiologia que atuam em serviços de atendimento em disfagia no estado de São Paulo. Os fonoaudiólogos foram convidados pessoalmente ou via correio eletrônico a responder ao questionário da pesquisa. Foi determinado como critérios de inclusão: ser fonoaudiólogo, atuar em serviços de atendimento em disfagia e concordar com o termo de consentimento. Como critério de exclusão foi determinado o não cumprimento dos critérios anteriores e o não preenchimento do questionário enviado. Os questionários respondidos retornaram à pesquisadora via correio, correio eletrônico ou entregues pessoalmente.

Os questionários aplicados abordaram: exemplos de alimentos utilizados nos procedimentos com pacientes disfágicos e a terminologia utilizada para designá-los.

\section{Procedimentos de Análise dos Dados}

As respostas dos questionários foram organizadas e tabuladas de acordo com os objetivos de cada questão.

As preparações referidas nos questionários foram reproduzidas e analisadas em triplicata, no Laboratório de Técnica Dietética da Universidade federal de Alfenas, por meio do Reômetro Brookfield com spindles $61,62,63$ e 64 na taxa de cisaIhamento de 30rpm, similar ao movimento de mastigação, sob temperatura de $25 \mathrm{C}^{\circ}$ (3).

A reprodução permitiu classificar os alimentos por meio da medição de suas unidades de viscosidade (centipoises - $\mathrm{cP}$ ), estabelecendo medidas quantitativas objetivas para a análise dos dados, agrupando as diversas preparações em faixas objetivas de padronização.

Os termos utilizados pelos profissionais para nomear cada nível de classificação dos alimentos foi especificado, verificando o número de termos propostos para cada nível, bem como as semeIhanças e diferenças entre os termos.

As preparações foram categorizadas segundo a proposta australiana de classificação(13) e em seguida os termos observados no presente estudo foram comparados com a proposta dos profissionais da Austrália.

O presente estudo foi aprovado pela Comissão de Ética em Pesquisa da Faculdade de Ciências Médicas da Universidade Estadual de Campinas sob protocolo n. 236/2008. 
Os dados foram analisados de forma estatística descritiva devido ao número restrito da amostra.

\section{RESULTADOS}

Foram analisados 18 questionários de fonoaudiólogos. Os dados referentes à caracterização dos sujeitos encontram-se na Tabela 1:
Foi questionado se, em algum momento da prática clínica, os fonoaudiólogos encontraram dificuldades no encaminhamento de pacientes aos demais profissionais devido a não uniformização da nomenclatura dos alimentos. As respostas apontaram (Figura 1):

A viscosidade das preparações mencionadas foi definida e classificada de acordo com a proposta australiana (Tabela 2).

Tabela 1 - Caracterização dos fonoaudiólogos de acordo com tempo e local de atuação, estágios de vida e tipo de disfagia atendida

\begin{tabular}{cccc}
\hline PERFIL DOS PROFISSIONAIS & $\mathbf{N}$ & $\%$ \\
\hline \multirow{2}{*}{ Tempo de Atuação } & 1 a 5 anos & 6 & 33,3 \\
& 5 a 10 anos & 4 & 22,2 \\
& mais de 10 anos & 8 & 44,4 \\
\hline \multirow{2}{*}{ Local de Atuação } & Hospitais & 7 & 38,8 \\
& Hospitais Escola & 11 & 61,1 \\
\hline \multirow{2}{*}{ Estágio de Vida } & Home Care & 4 & 22,2 \\
& RN e crianças & 7 & 38,8 \\
& Adultos e idosos & 13 & 72,2 \\
\hline \multirow{2}{*}{ Disfagia Atendida } & Mecânica & 9 & 50 \\
& Neurogênica & 14 & 77,7 \\
\hline
\end{tabular}

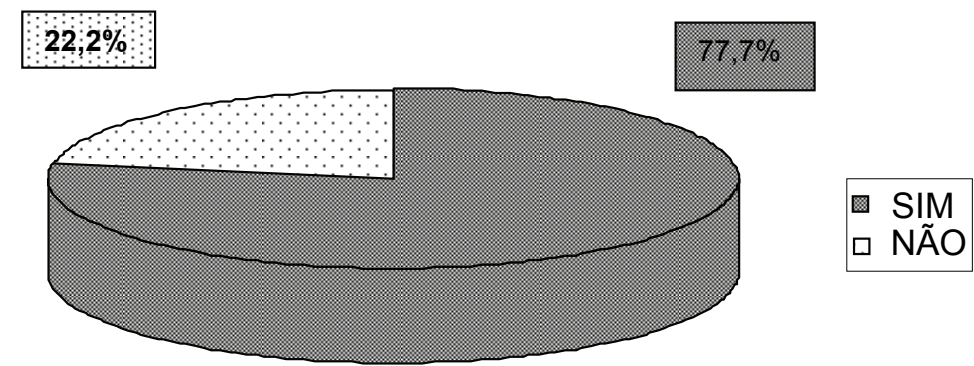

Figura 1 - Dificuldades em encaminhamentos pela ausência de padronização da nomenclatura

Tabela 2 - Análise do comportamento reológico e classificação dos alimentos segundo o padrão australiano

\begin{tabular}{ccc}
\hline PREPARAÇÃO & CLASSIFIC. AUSTRÁLIANA & VISCOSIDADE (Cp) \\
\hline Água & Líquido & 2 \\
Suco de manga & Nível 150 - Ligeiramente espesso & 150 \\
logurte & Nível 400 - Moderadamente espesso & 800 \\
Danoninho & Nível 900 - Extremamente espesso & 8400 \\
Danete & Nível 900 - Extremamente espesso & 13000 \\
Mingau & Nível 900 - Extremamente espesso & 2000 \\
Sopa de legumes batida & Nível 900 - Extremamente espesso & 3000 \\
Purê & Nível 900 - Extremamente espesso & 4000 \\
Pudim & Alimento macio & 4800 \\
Sopa amassada & Alimento macio & 7000 \\
Bolacha amolecida no leite & Alimento macio & \\
Arroz, feijão, pão, carne & Alimentos não modificados & \\
\hline
\end{tabular}


A terminologia utilizada pelos fonoaudiólogos para designar as diferentes viscosidades foi comparada com a terminologia sugerida pela proposta australiana, tendo por critério de referência a preparação dos alimentos (Tabela 3 ).

É possível observar que a nomenclatura utilizada pelos fonoaudiólogos é comum nos dois extremos da escala (líquido e sólido) sendo que as demais consistências apresentam-se comumente como graduações de líquido e pastoso ou ainda descrições de características específicas (fino, grosso, médio, homogêneo, heterogêneo).

Tabela 3 - Terminologia utilizada pelos fonoaudiólogos para classificação dos alimentos em comparação com a proposta australiana

\begin{tabular}{|c|c|c|c|}
\hline \multirow{2}{*}{ PREPARAÇÕES } & \multicolumn{2}{|c|}{ TERMINOLOGIA } & \multirow{2}{*}{ TOTAL } \\
\hline & AUSTRALIANA & FONOAUDIÓLOGOS & \\
\hline \multirow{3}{*}{$\begin{array}{l}\text { Água, } \\
\text { Suco }\end{array}$} & Líquido & Líquido & 7 \\
\hline & & Líquido ralo & 2 \\
\hline & & Líquido Fino & 2 \\
\hline \multirow{7}{*}{$\begin{array}{l}\text { Suco de } 2 \text { frutas } \\
\text { Suco de manga } \\
\text { Suco de mamão } \\
\text { logurte }\end{array}$} & Ligeiramente & Líquido Engrossado & 5 \\
\hline & Espesso & Líquido Pastoso & 3 \\
\hline & & Líquido Pastoso Homogêneo & 1 \\
\hline & & Líquido Grosso & 1 \\
\hline & & Pastoso Fino & 1 \\
\hline & & Vitamina & 1 \\
\hline & & Bliss & 1 \\
\hline \multirow{9}{*}{$\begin{array}{l}\text { Sopa Liquefeita } \\
\text { Vitamina }\end{array}$} & Moderadamente & Líquido Engrossado & 1 \\
\hline & Espesso & Líquido Pastoso Homogêneo & 1 \\
\hline & & Líquido Pastoso & 2 \\
\hline & & Pastoso Homogêneo Fino & 1 \\
\hline & & Pastoso Fino & 5 \\
\hline & & Pastoso Homogêneo & 1 \\
\hline & & Vitamina & 1 \\
\hline & & Danete & 1 \\
\hline & & Pastoso & 4 \\
\hline \multirow{8}{*}{$\begin{array}{l}\text { Sopa Batida } \\
\text { Mingau } \\
\text { Purê de Legumes }\end{array}$} & Extremamente & Pastoso Homogêneo & 3 \\
\hline & Espesso & Pastoso Grosso & 3 \\
\hline & & Pastoso Homogêneo Grosso & 1 \\
\hline & & Pastoso Engrossado & 1 \\
\hline & & Pastoso Médio & 1 \\
\hline & & Mingau & 1 \\
\hline & & Danoninho & 1 \\
\hline & & Semi-sólido pastoso & 1 \\
\hline \multirow{6}{*}{$\begin{array}{l}\text { Sopa com pedaços } \\
\text { Massas }\end{array}$} & Alimentos & Pastoso grosso & 1 \\
\hline & Macios & Pastoso Heterogêneo & 3 \\
\hline & & Pastoso Engrossado & 1 \\
\hline & & Sólido Amassado & 1 \\
\hline & & Semi-sólido & 1 \\
\hline & & Sólido & 1 \\
\hline Carne Moída & Alimentos Picados e & Pastoso Grosso & 1 \\
\hline \multirow[t]{4}{*}{ Frango Desfiado } & Úmidos & Sólido Amassado & 1 \\
\hline & & Sólido Macio & 1 \\
\hline & & Semi-sólido & 3 \\
\hline & & Sólido & 2 \\
\hline Arroz & Alimentos não & Sólido & 7 \\
\hline Feijão & modificados & Sólido Duro & 1 \\
\hline Carne & & Dieta Geral & 1 \\
\hline
\end{tabular}




\section{DISCUSSÃO}

No que se refere à caracterização da amostra o tempo de atuação dos profissionais na área de disfagia variou nas três classificações propostas, apresentando maior concentração entre 1 e 5 anos e acima de 10 anos de experiência na área. $\mathrm{O}$ número de participantes com 1 a 5 anos de experiência reflete um possível crescimento da demanda em disfagia na Fonoaudiologia.

Quanto ao local de atuação, a maioria dos fonoaudiólogos participantes atua em hospitais escola. A maior concentração de profissionais atuantes em locais de ensino justifica-se por serem tais hospitais os principais serviços de atendimento em disfagia no estado de São Paulo, além de terem como característica um maior foco em pesquisas, o que justifica sua participação no estudo.

A população atendida pelos profissionais participantes mostrou-se heterogênea (Tabela 1). Tal dado evidencia a necessidade do aumento do número de equipes fonoaudiológicas especializadas nos hospitais.

No que se refere a possíveis dificuldades geradas pela ausência de uma nomenclatura padrão, catorze fonoaudiólogos $(77,7 \%)$ apontaram problemas nos encaminhamentos a demais profissionais. Tais dados indicam que o tema em discussão apresenta relevância para a prática clínica em disfagia uma vez que tem trazido dificuldades. Quatro fonoaudiólogos não referiram dificuldades nos encaminhamentos, porém três deles destacaram que descrevem os alimentos orientados para evitar mal entendidos, fator que evidencia preocupação dos mesmos na troca de informações com os colegas e na orientação ao paciente.

A classificação do comportamento reológico permitiu observar que a maioria dos participantes faz uso de parâmetros alimentares similares em sua prática clínica, porém apresentam diferenças na forma de nomeação de uma mesma preparação.

Foi possível observar a similaridade entre os alimentos citados devido à avaliação física da viscosidade. A medição dos líquidos engrossados possibilitou sua classificação em faixas de viscosidade, identificando dessa forma um de seus parâmetros reológicos ${ }^{14,15}$. A classificação mostrou líquidos engrossados de viscosidades próximas, indicando que os profissionais utilizam alimentos com características reológicas similares em sua prática, embora os nomeiem de formas diferentes.

Para nomear sete diferentes classes de alimentos, segundo a proposta australiana ${ }^{13}$, os participantes utilizaram 33 termos diferentes. A maior diversidade de termos ocorreu para a nomeação dos líquidos engrossados os quais foram apresentados por meio de 09 graduações do termo 'líquido' e 10 do termo 'pastoso', além de 06 termos descritivos. Tais dados são semelhantes aos estudos internacionais 12,16-18 com diversidade de termos para a nomeação de um mesmo alimento nas fases anteriores a padronização da nomenclatura.

Os líquidos engrossados constituem uma categoria ampla, em que pequenas mudanças de viscosidade provocam alterações no processo de deglutição que podem significar uma deglutição segura ou insegura ao paciente ${ }^{9,19}$. Dessa forma, cabe ao profissional especificar detalhadamente a que tipo de liquido engrossado está se referindo e certificarse que seu interlocutor, seja outro profissional ou cuidador, compreendeu sua orientação.

Quanto aos termos utilizados, os fonoaudiólogos citaram principalmente líquido, pastoso e sólido, conforme observado na Tabela 3. Os demais termos são, em sua maioria, graduações dos termos principais (líquido engrossado, pastoso fino, semi-sólido) ou ainda especificações do alimento (pastoso homogêneo ou heterogêneo). A estratégia de nomeação utilizada baseia-se na descrição da espessura dos líquidos, ou seja, utilizam graduações de um termo específico que evidenciam seu grau de espessamento.

Essa estratégia de nomeação é a mesma utilizada pela proposta australiana de padronização da nomenclatura ${ }^{13}$, a qual apresenta um termo principal, no caso 'espesso', seguido de graduações (ligeiramente, moderadamente, extremamente) para classificação dos líquidos modificados.

Os fonoaudiólogos do estudo apresentaram ainda 06 termos (cremoso, 'danete', 'danoninho', vitamina, 'bliss' e mingau) que evidenciam como estratégia de nomeação a descrição/comparação do alimento em questão com outro de conhecimento geral. Tal estratégia aproxima-se da utilizada pela proposta americana de classificação de líquidos modificados ${ }^{12}$ a qual também se baseia na comparação/descrição ao utilizar os termos ralo, néctar, mel e pudim, podendo ser entendidos no pareamento proposto na classificação como: 'líquidos como mel' ou 'líquidos parecidos com mel', por exemplo.

A estratégia de nomeação baseada na comparação de alimentos com outros de conhecimento geral apresenta-se como uma forma de fácil compreensão no momento de orientação da dieta. A comparação entre texturas facilita a visualização de possíveis alimentos similares o que auxilia o processo de orientação aos cuidadores, na medida em que o profissional pode se referir a alimentos do cotidiano dos mesmos. 
Optou-se neste estudo pelo uso da proposta australiana ${ }^{13}$ de classificação dos alimentos por essa proposta ser interdisciplinar (consenso entre fonoaudiólogos e nutricionistas australianos) e apresentar um número de subdivisões e estratégia de nomeação compatíveis com as apresentadas pelos profissionais participantes deste estudo. Cabe destacar que a proposta veio ao encontro das necessidades metodológicas desse estudo, porém, no momento da orientação ao paciente, a mesma parece apresentar-se pouco didática, gerando maior dificuldade para compreensão pelos cuidadores por utilizar termos pouco usuais, sendo necessário fazer uso constante de exemplos de alimentos para garantir a compreensão de seus termos.

Considerando que não há estabelecido um padrão de nomenclatura no país, o uso de exemplos de alimentos cotidianos facilita a compreensão da orientação não só aos cuidadores, mas também entre os diversos profissionais atuantes no caso. É necessário certificar-se no momento da orientação aos cuidadores que a linguagem utilizada é clara, que a interpretação dos termos utilizados corresponde a idéia do profissional. Nessas situações, o uso de termos específicos pode dificultar a compreensão do usuário do serviço, sendo necessário adequar a linguagem por meio de termos cotidianos, sendo o uso de exemplos uma das estratégias mais seguras para garantir a compreensão dos cuidadores.

A proposta americana de classificação dos alimentos ${ }^{12}$ pode ser utilizada como um instrumento positivo no momento da orientação uma vez que seus diferentes níveis de classificação de líquidos engrossados podem ser facilmente demonstrados visualmente, o que a torna mais didática, ao permitir que o cuidador estabeleça relações visuais entre diferentes alimentos de seu cotidiano que apresentam viscosidade ou texturas similares.

Internacionalmente iniciam-se discussões para o estabelecimento de padrões de nomenclatura entre países ${ }^{5}$. Dessa forma, são necessários maiores estudos na área para que em um futuro próximo possa se estabelecer um consenso entre os profissionais no Brasil, possivelmente baseando a classificação utilizada no país em uma adaptação de propostas já existentes buscando assim futura concordância com padrões internacionais.

Os resultados do presente estudo indicaram que o tema em discussão é de grande relevância para a intervenção em disfagia, pois tem trazido dificuldades tanto em seus aspectos clínicos quanto acadêmicos. Reforça-se, dessa forma, a necessidade de estabelecer uma nomenclatura comum em consenso não apenas entre fonoaudiólogos mas com os diversos profissionais da equipe de intervenção ${ }^{20}$, especialmente os que atuam mais diretamente com a dieta dos pacientes (fonoaudiólogos, nutricionistas, equipe de enfermagem). Há necessidade de ampliar as discussões para que no futuro possa se estabelecer uma nomenclatura unificada no país.

\section{CONCLUSÕES}

Os fonoaudiólogos participantes do estudo, em sua maioria, referiram fazer uso em sua prática clínica de alimentos com características reológicas próximas;

Fonoaudiólogos de diferentes serviços de atenção em disfagia utilizam diferentes terminologias para designar a mesma preparação;

Os fonoaudiólogos participantes utilizaram 33 termos para descrever 07 níveis de classificações de alimentos. 


\begin{abstract}
Purpose: to study the aspects of rheological behavior and labels of texture-modified foods and thickened fluids as used by teams of speech-language pathologist of dysphasia's services of São Paulo based on Australian standardized labels and definitions and rheological behavior. Method: quantitative and descriptive study. Data were collected from semi-structured questionnaires with speech-language pathologists in dysphasia teams. The questions were about foods used in clinical practice and the form of classification, and included the number of levels and labels used, and information on the interdisciplinary performance. The viscosities were measured in centipoises (cP) and the foods and fluids were classified according to rheological behavior. The last step was food classification, professionals reported using foods of the same rheological behavior in their practices. Results: speech-language pathologists related 33 different labels to 07 levels of food textures and fluid thickness based on Australian standardized. The main topics were: liquid, pasty and solid, followed by grades and specifications. Speech pathologists from different dysphasia care services use different terminologies in order to designate the same preparation in the Sate of São Paulo. Conclusion: larger studies are needed to enable us to set out unified terminology in Brazil.
\end{abstract}

KEYWORDS: Deglutition Disorders; Rheology; Interdisciplinary Communication

\section{REFERÊNCIAS}

1. Sopade PA, Halley PJ, Cichero JAY, Ward L. Rheological characterisation of food thickeners marketed in Australia in various media for the management of dysphagia. I: Water and cordial. Journal of Food Engineering. 2006; 79 (1): 69-82.

2. Sopade PA, Halley PJ, Cichero JAY, Ward L, Hui LS, Teo K. Rheological characterisation of food thickeners marketed in Australia in various media for the management of dysphagia. II: Milk as a dispersing medium. Journal of Food Engineering. 2008;84 (4): 553-62.

3. Silva LBC, Ikeda CM. Cuidado nutricional na disfagia: uma alternativa para maximização do estado nutricional. Ver. Brás. Nutr. Clin. 2009; 24: 203-10.

4. Silva FC, Guimarães DHP, Gasparetto CA. Reologia do suco de acerola: efeitos da concentração e temperatura. Ciênc. Tecnol. Aliment. 2005; 25(1): 121-6.

5. Cichero JAY. Rheology and Dysphagia: a partnership out of Pandora's Box. In: Dysphagia Reseach Society: Annual Meeting - Annals. New Orleans, 2009.

6. Logemann JA. Dysphagia: Evaluation and Treatment. Folia Phoniatr Logop. 1995; 47: 121-9.

7.Costa MMB. Anatomia Funcional da Fase Oral da Deglutição. Deglutição e Disfagia (Conceitos Básicos) - Material Instrucional ICB-UFRJ; 2005: 29-41.
8. Steele CM, Van Lieshout PHHM. LingualMandibular Coordination During Liquid Swallow. Dysphagia. 2008; 23(1): 33-46.

9. Troche MS, Sapienza CM, Rosenbek JC. Effects of Bolus Consistency on Timing and Safety of Swallow in Patients with Parkinson_s Disease. Dysphagia. 2008; 23(1):26-32.

10. Saitoh E, Shibata S, Koichiro M, Baba M, Fujii W, Palmer JG. Chewing and food C consistency: effects on bolus transport and swallow initiation. Dysphagia. 2007; 22:100-7.

11. British Dietetic Association. National Descriptors for Texture Modification in Adults. The British Dietetic Association, Royal College of Speech and Language Therapists 2002 (May).

12. American Speech-Language-Hearing Association. Roles of speechlanguage pathologists in swallowing and feeding disorders: technical report. ASHA Desk Reference. 2002; 3:181-99.

13. Atherton M, Bellis-Smith N,Cichero JAY, Suter M. Texture-modified foods and thickened fluids as used for individuals whith dysphagia : Australian standardized labels and definitions. Nutrition and Dietetics 2007; 64 (2):S53-S76.

14. Steele CM, Cichero JAY. A Question of Rheological Control. Dysphagia (2008) 23:199-201. 15 Garcia JM, Iv EC, Matta Z, Clark M. Serving Temperature Viscosity Measurements of Nectarand Honey-Thick Liquids. Dysphagia; 2008; 23 (1):65-75.

16. Penman JP, Thomson M. A review of the textured diets developed for the management of dysphagia. J Hum Nutr Diet 1998; 11: 51-60. 
17. Cichero JAY, Hay G, Jackson O, Halley PJ, Murdoch BE. Which of these is not like the others? An inter-hospital study of the viscosity of thickened fluids. J Speech Hear Res 2000; 43: 537-47.

18. Cichero JAY, Hay G, Murdoch BE et al. How thick is thick? A multi-centre study of the rheological and material property characteristics of meal-time fluids and videofluoroscopy. Nutrition \& Dietetics 2007; 64 (Suppl. 2): S53-S76.
19. Groher ME, Crary MA, Carnaby (Mann) GD, Vickers Z, Aguilar C. The impact of rheologically controlled materials on the identification of airway compromise on the clinical and videofluoroscopic swallowing examinations. Dysphagia 2006;21: 218-25.

20. Clavé $P$, Terré $R$, de Kraa $M$, Serra $M$. Approaching oropharyngeal dysphagia. Rev Esp Enferm Dig 2004; 96: 119-31.

http://dx.doi.org/10.1590/S1516-18462011005000145

RECEBIDO EM: 15/03/2011

ACEITO EM: 23/06/2011

Endereço para correspondência:

Marina de Sordi

Avenida Olívio Boa, 380 - bloco 05 - apto. 32

Jundiaí, SP

CEP: 13214-550

E-mail: marina_fono01@yahoo.com.br 